\title{
Reversals of Acoustic Radiation Torque in Bessel Beams Using Theoretical and Numerical Implementations in Three Dimensions
}

\author{
Zhixiong Gong, ${ }^{1,2, \dagger}$ Philip L. Marston, ${ }^{2}$ and Wei $\mathrm{Li}^{1, *}$ \\ ${ }^{1}$ School of Naval Architecture and Ocean Engineering, Huazhong University of Science and Technology, \\ Wuhan 430074, China \\ ${ }^{2}$ Department of Physics and Astronomy, Washington State University, Pullman, Washington 99164-2814, USA
}

(Received 10 January 2019; revised manuscript received 1 May 2019; published 11 June 2019)

\begin{abstract}
Acoustic radiation torque (ART) plays an important role in the subject of acoustophoresis, which could induce the spinning rotation of particles on their own axes. The partial wave series method (PWSM) and T matrix method (TMM) are employed to investigate the three-dimensional radiation torques of both spherical and nonspherical objects in a single Bessel beam over broader frequencies (from Rayleigh scattering to geometric-optics regimes), with emphasis on the parametric conditions for torque reversal and the corresponding physical mechanisms. For elastic objects, the dipole, quadrupole, and even several higherscattering modes are dominant to induce the axial ARTs beyond the Rayleigh limit with a relatively large offset from the particle centroid to the beam axis in Bessel beams. The reversals appear with parametric conditions (including wave number, cone angle, and offset) for the extrema or null values of the corresponding cylindrical Bessel functions. The reversal of transverse ART from a rigid nonspherical particle is also investigated in an ordinary Bessel beam and the geometrical surface roughness is briefly studied for the effect on the radiation torques. This suggests the possibility of acoustic tweezers controlling the spinning motion of particles within and beyond the Rayleigh limit.
\end{abstract}

DOI: 10.1103/PhysRevApplied.11.064022

\section{INTRODUCTION}

Acoustic manipulations have attracted more and more attention for the peculiar advantages of biocompatibility and remote contact, which mainly consider the translocation and rotation dynamics of the particles immersed in fluids. As compared to its optical counterpart, acoustic tweezers could provide larger forces and torques with the same incident wave field intensity and get rid of heating issues [1]. Meanwhile, the transducers in designing the acoustic tweezers could provide a broader band of frequencies from $\mathrm{kHz}$ to $\mathrm{GHz}$, which could be applied for a large range of particle sizes [2]. It is noteworthy that the acoustic radiation force (ARF) is induced by the transfer of linear momentum, while the acoustic radiation torque (ART) comes from the exchange of angular momentum from the acoustic field to immersed particles. In general, acoustic scattering [3-11] and streaming [12-16] are the two typical kinds of physical mechanisms to move or rotate particles in the fluids illuminated by vortex beams [17-22] or standing waves [23-27]. For the ARFs in a viscous fluid, the acoustic streaming will dominate for small-sized particles,

\footnotetext{
*hustliw@hust.edu.cn

†Present address: Univ. Lille, CNRS, Centrale Lille, ISEN, Univ. Polytechniques Hauts-de-France, UMR 8520-IEMN, International laboratory LIA/LICS, F-59000 Lille, France.
}

whereas the acoustic scattering plays a dominant role for particles with sizes larger than the critical value [28-30]. To date, the ARF has been studied extensively in the field of particle manipulation including particle trapping, selectivity, sorting, patterning, orbital rotation, and so on, while the applications of spinning (i.e., rotation around the particle centroid) induced by ART still need more attention, especially on the reversal conditions and physical mechanisms for particles with different geometrical shapes and material composition. In addition, most of the theoretical work related to acoustic torque are focused on the Rayleigh limitation [31]. It is worthwhile noting that particle dynamics are interesting and promising beyond long-wavelength approximation, such as in the Mie and geometric-optics regimes. Hence, it is necessary to develop physical models and theoretical or numerical methods to take the broadband frequency analysis in acoustofluidics into consideration.

Standing waves and vortex beams are very promising candidates in designing acoustic tweezers for biomedical applications and material chemistry in the future, which could both induce the three-dimensional ARFs to move or trap particles and the ARTs to spin particles in the acoustic field under certain circumstances. The vortex beams could be employed to rotate the particles by the transfer procedure of the orbital angular momentum between the acoustic field and particles. Rotations give extra degrees of particle dynamics other than the translocations in three 
dimensions. For particles in a single vortex beam, the relative location of the object and beam axis will affect the ARFs and ARTs exerted on the object. Zhang and Marston derived a theoretical relationship between the axial torque $T_{z}$ on an axisymmetric object located on the axis of a single vortex beam and the absorption energy $P_{\text {abs }}$, weighting by the ratio of topological charge $M$ and wave angular frequency $\omega$, written as $T_{z}=(M / \omega) P_{\text {abs }}$ [32]. However, this theory cannot be applied when the particle moves off the beam axis. In the following work by Zhang, a similar expression was given for the off-axis situation [31], which is, in fact, based on the Graf's addition theorem of Bessel functions [33] to use the summation of on-axis torques to describe the off-axial condition. Under this circumstance, the sign of effective topological charge could not be decided intuitively since it depends on the weighting amplitude and cylindrical Bessel functions. As observed in the aforementioned formulas for the on-axis situation, the direction of axial ART can be determined once the signs of the topological charge are known. The sign of the effective topological charge is very important for finding the positive or negative axial torques as well as the reversal parameters. Zhang gave the theoretical derivation of the effective topological charge for a small particle (i.e., long-wavelength approximation) and found the condition for axial torque reversal in the off-axis incidence [31]. It is noteworthy that the ARTs from small particles of fluid [34] and viscoelastic [35] materials have been investigated in the traveling/standing plane wave and Bessel beam with the on-axis configuration. The secondary radiation torques from multiple particles in arbitrary position have been studied in the Mie scattering regime under the illumination of traveling and standing plane waves [36]. However, it still needs to be discussed for a more general case in Bessel beams, which will include a broadband frequency (in and beyond the Rayleigh regime). This is discussed and investigated in the present work.

On the other hand, Baresch et al. recently found that the quadrupolar particle vibration mode could not be neglected even in the Rayleigh regime for the viscous dissipation in the fluid and absorption in the particle [37]. Hence, it is important to compute the total radiation torque at a broadband frequency, which will consider not only the dipolar vibration mode, but also higher-order vibration modes. The partial wave series method (PWSM) is one kind of theoretical method that considers the necessary individual vibration modes and can correctly compute the scattering field of particles immersed in fluid. Based on the angular momentum stress tensor theory, the ARTs could be expressed in terms of the incident and scattered beam shape coefficients with the explicit derivation given below. It is anticipated that the PWSM could provide a theoretical method for spherical shapes to calculate the exact torques induced by the acoustic field. In biomedical applications and physical chemistry, the spherical shapes cannot always model the particle properly. Hence, the model using more accurate geometric shapes will predict more precise torques on the particles of interest. To this end, a versatile numerical method, the T matrix method (TMM) [38-41], is introduced to calculate the three-dimensional ARTs for the nonspherical particle. Note that the TMM could apply for a spherical shape as a special case of general three-dimensional shapes. In addition, it is necessary to pay attention to the surface shape of the particle since the geometrical shape will influence the scattering field and accordingly the transfer process of both the linear and angular momenta between the particle and the acoustic field. The influence of the surface roughness will be briefly studied in this work. In combination with the work of ARFs in three dimensions [11], it is promising to develop the acoustic tweezers numerical toolbox with six degrees of freedom $[42,43]$, which could provide precise and stable manipulations and will finally facilitate the applications of acoustofluidics technology in ultrasound diagnosis and clinical medicine. The orbital rotation of the particle around the beam axis (due to the lateral ARFs) is outside the scope of this work, while the emphasis will be on the spin rotation around the particle center induced by the ARTs.

\section{PHYSICAL MODELS AND MATHEMATICAL FORMULAS}

\section{A. Three-dimensional ARTs in arbitrary acoustic waves}

The theoretical methods (partial wave series method) and the numerical method ( $\mathrm{T}$ matrix method) have been demonstrated for acoustic scattering in our previous work [3-5,33,39-44], which will not be shown here for simplicity. Both the PWSM and TMM provide a linear relationship between the incident $\left(a_{n m}\right)$ and scattered $\left(s_{n m}\right)$ beam shape coefficients. In the following, the acoustic radiation torques in three dimensions are independently derived based on the radiation angular momentum stress tensor approach $[32,45,46]$, which gives a general relationship between the three-dimensional torques and (incident and scattered) beam shape coefficients.

Consider an object surrounded by an ideal fluid (the angular momentum stress tensor meets $\nabla \cdot \mathbf{S}_{T}^{\prime}=0$ ) illuminated by an arbitrary acoustic field and based on the divergence theorem, the integral of the angular momentum stress tensor over the object surface $(S)$ could be extended to an arbitrary spherical surface in the far-field $\left(S_{0}\right.$ with $r \rightarrow \infty$ ) with the center located at the geometric center of the particle $O$ (see Fig. 1). This procedure facilitates the theoretical and numerical methods to compute the ARTs on arbitrary shape objects and gives a uniform formula in terms of incident and scattered fields. According to the formula $\mathbf{T}=\iint_{S_{0}} \mathbf{S}_{T}^{\prime} d \mathbf{S}$ and the angular momentum stress 


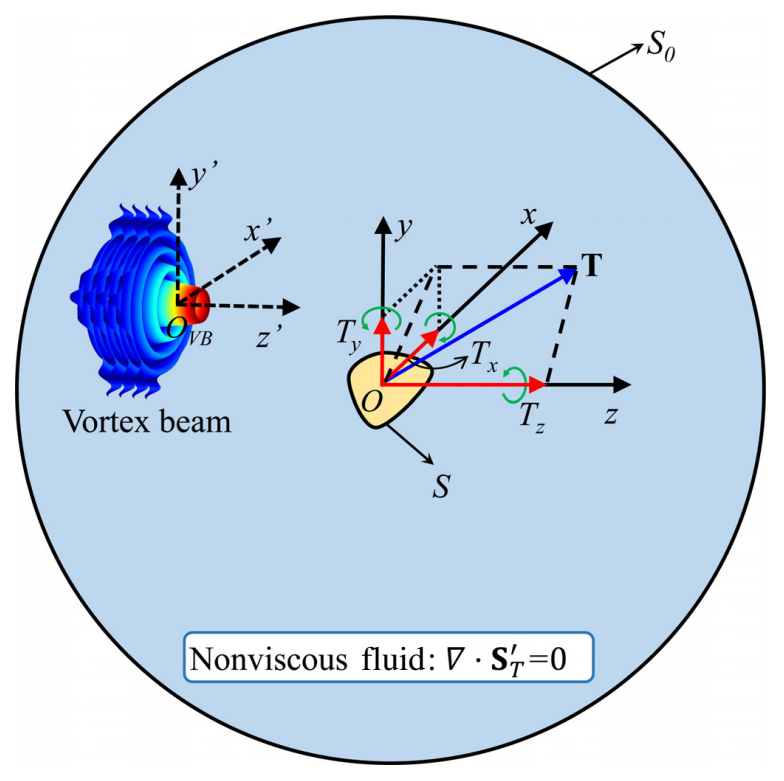

FIG. 1. Schematic of an arbitrarily shaped object in a nonviscous fluid illuminated by a Bessel beam with arbitrary topological charge and location.

tensor $\mathbf{S}_{T}^{\prime}=\mathbf{r} \times \mathbf{S}_{T}$ where $\mathbf{S}_{T}=\langle L\rangle-\rho_{0}\langle\mathbf{u u}\rangle$ is the linear momentum stress tensor, the torque vector could be expressed as

$$
\mathbf{T}=-\rho_{0} \iint_{S_{0}}\langle L\rangle \mathbf{r} \times d \mathbf{S}-\rho_{0} \iint_{S_{0}}\langle(\mathbf{r} \times \mathbf{u}) \mathbf{u}\rangle d \mathbf{S}
$$

where $\rho_{0}$ is the fluid density, $\langle L\rangle$ is the time average of the Lagrangian density, and $\mathbf{u}$ denotes the total velocity vector. The differential surface area in the far field is $d \mathbf{S}=\mathbf{n} \cdot r^{2} \sin \theta d \theta d \varphi$ with $\mathbf{n}$ the outward unit normal vector. Introducing the relationship $\mathbf{r} \times d \mathbf{S}=0$ and the time average of complex numbers, Eq. (1) could be simplified as

$$
\mathbf{T}=-\frac{\rho_{0}}{2} \operatorname{Re} \iint_{S_{0}}\left\{(\mathbf{r} \times \nabla \Phi) \cdot \mathbf{n} \nabla \Phi^{*}\right\} d S,
$$

where $\Phi$ is the total velocity potential having $\mathbf{u}=\nabla \Phi$ and $*$ denotes complex conjugation. For convenience, the angular momentum operator $\mathbf{L}$ from quantum mechanics is introduced with $\mathbf{L}=-i(\mathbf{r} \times \nabla)$ [47], where $i$ is the imaginary unit. By substituting $\mathbf{r} \times \nabla=i \mathbf{L}$ and $\Phi=\Phi_{i}+\Phi_{s}$ into Eq. (2), it is rearranged as

$$
\begin{aligned}
\mathbf{T}= & \frac{\rho_{0}}{2} \operatorname{Im} \iint_{S_{0}}\left\{\frac{\partial \Phi_{i}{ }^{*}}{\partial r} \mathbf{L} \Phi_{i}+\frac{\partial \Phi_{i}{ }^{*}}{\partial r} \mathbf{L} \Phi_{s}+\frac{\partial \Phi_{s}{ }^{*}}{\partial r} \mathbf{L} \Phi_{i}\right. \\
& \left.+\frac{\partial \Phi_{s}{ }^{*}}{\partial r} \mathbf{L} \Phi_{s}\right\} d S
\end{aligned}
$$

where Im designates the imaginary part. The far-field incident $\Phi_{i}$ and scattered $\Phi_{s}$ potentials are, respectively

$$
\begin{aligned}
& \Phi_{i}=\Phi_{0} \sum_{n=0}^{\infty} \sum_{m=-n}^{n} a_{n m} j_{n}(k r) Y_{n m}(\theta, \varphi) \\
& \Phi_{s}=\Phi_{0} \sum_{n=0}^{\infty} \sum_{m=-n}^{n} s_{n m} h_{n}^{(1)}(k r) Y_{n m}(\theta, \varphi) .
\end{aligned}
$$

In the above, $a_{n m}$ and $s_{n m}$ are the incident and scattered coefficients of expansion (beam shape coefficients), $\Phi_{0}$ is the beam amplitude, and $j_{n}(k r)$ and $h_{n}^{(1)}(k r)$ are the spherical Bessel and Hankel functions of the first kind, respectively, $Y_{n m}(\theta, \varphi)$ represents the normalized spherical harmonics and $k$ is the wave number. In the absence of scatterers there is no transfer of angular momentum in the ideal fluid, and therefore, the incident acoustic field will remain as original so that the term in Eq. (3) only involving $\Phi_{i}$ will vanish. In order to further simplify the ART in Eq. (3) and use incident and scattered beam shape coefficients to describe the ART, the far-field $(k r \rightarrow \infty)$ asymptotic expressions of the spherical Bessel function and Hankel function of the first kind with a recursion relation of the spherical Bessel function and its derivative are employed

$$
\begin{aligned}
j_{n}(k r) & \simeq i^{-(n+1)} e^{i k r} / 2 k r+i^{n+1} e^{-i k r} / 2 k r, \\
h_{n}^{(1)}(k r) & \simeq i^{-(n+1)} e^{i k r} / k r, \\
j^{\prime}{ }_{n}(k r) & =(n / k r) j_{n}(k r)-j_{n+1}(k r) .
\end{aligned}
$$

By inserting Eqs. (4)-(6) into Eq. (3) and conducting several algebraic operations, the ART vector can be described briefly in terms of the incident and scattered beam shape coefficients, such that

$$
\begin{aligned}
\mathbf{T}= & -\frac{\rho_{0} \Phi_{0}^{2}}{2 k r^{2}} \operatorname{Re} \iint_{S_{0}}\left\{\sum_{n m} \sum_{n^{\prime} m^{\prime}} i^{n-n^{\prime}}\left(a_{n m}^{*}+s_{n m}^{*}\right) s_{n^{\prime} m^{\prime}}\right. \\
& \left.\times\left(Y_{n m}^{*} \mathbf{L} Y_{n^{\prime} m^{\prime}}\right)\right\} d S .
\end{aligned}
$$

To obtain the ARTs theoretically or numerically, the outward unit normal vector $\mathbf{n}=\sin \theta \cos \varphi \mathbf{e}_{\mathbf{x}}+$ $\sin \theta \sin \varphi \mathbf{e}_{\mathbf{y}}+\cos \theta \mathbf{e}_{\mathbf{z}}$ in Cartesian coordinates is applied for the projections of ARTs in three dimensions. The dimensionless ART function $\boldsymbol{\Gamma}$ is introduced to depict the ART with the relationship $\mathbf{T}=\pi r_{0}^{3} \mathrm{I}_{0} c_{0}^{-1} \boldsymbol{\Gamma}$, where $I_{0}=$ $\left(\rho_{0} c_{0} / 2\right)\left(k \Phi_{0}\right)^{2}$ with $c_{0}$ being the speed of sound in the surrounding fluid and $r_{0}$ is the characteristic dimension of the target (i.e., the radius of the maximum circumscribed sphere). Finally, the dimensionless ART function $\boldsymbol{\Gamma}$ could 
be rewritten as

$$
\begin{aligned}
\boldsymbol{\Gamma}= & -\frac{1}{\pi\left(k r_{0}\right)^{3}} \operatorname{Re} \iint_{S_{0}}\left\{\sum_{n m} \sum_{n^{\prime} m^{\prime}} i^{n-n^{\prime}}\left(a_{n m}^{*}+s_{n m}^{*}\right) s_{n^{\prime} m^{\prime}}\right. \\
& \left.\times\left(Y_{n m}^{*} \mathbf{L} Y_{n^{\prime} m^{\prime}}\right)\right\} \sin \theta d \theta d \varphi .
\end{aligned}
$$

In Cartesian coordinates, the angular momentum operator is expressed as $\mathbf{L}=L_{x} \mathbf{e}_{x}+L_{y} \mathbf{e}_{y}+L_{z} \mathbf{e}_{z}$, with the three components [47]

$$
\begin{aligned}
& L_{x}=i \sin \varphi \frac{\partial}{\partial \theta}+i \cot \theta \cos \varphi \frac{\partial}{\partial \varphi}, \\
& L_{y}=-i \cos \varphi \frac{\partial}{\partial \theta}+i \cot \theta \sin \varphi \frac{\partial}{\partial \varphi}, \\
& L_{z}=-i \frac{\partial}{\partial \varphi} .
\end{aligned}
$$

To implement the integration of the product of the angular momentum operator and spherical harmonics over the solid angle, the ladder operators $L_{ \pm}=L_{x} \pm i L_{y}$ are considered, which are related to the spherical harmonics as $L_{ \pm} Y_{n m}=\sqrt{(n \pm m+1)(n \mp m)} Y_{n, m+1}$. In addition, the $z$ component $L_{z}$ of the angular momentum operator has a relationship with the spherical harmonics as $L_{z} Y_{n m}=$ $m Y_{n m}$. Substituting the relationships of the angular momentum operator and spherical harmonics into Eq. (8), the dimensionless ARTs in Cartesian coordinates could be derived as follows:

$$
\begin{aligned}
\Gamma_{x}= & -\frac{1}{2 \pi\left(k r_{0}\right)^{3}} \operatorname{Re} \sum_{n m}\left(a_{n m}^{*}+s_{n m}^{*}\right)\left(b_{n m-} s_{n, m-1}\right. \\
& \left.+b_{n m+} s_{n, m+1}\right), \\
\Gamma_{y}= & -\frac{1}{2 \pi\left(k r_{0}\right)^{3}} \operatorname{Im} \sum_{n m}\left(a_{n m}+s_{n m}\right)\left(b_{n m+} s_{n, m+1}^{*}\right. \\
& \left.-b_{n m-} s_{n, m-1}^{*}\right), \\
\Gamma_{z}= & -\frac{1}{\pi\left(k r_{0}\right)^{3}} \operatorname{Re} \sum_{n m} m\left(a_{n m}^{*}+s_{n m}^{*}\right) s_{n^{\prime} m^{\prime}},
\end{aligned}
$$

with coefficients defined as $b_{n m-}=\sqrt{(n-m+1)(n+m)}$ and $b_{n m+}=\sqrt{(n+m+1)(n-m)}$. Re designates the real part of the indicated expression. It is noteworthy that the above formulas, which are derived independently, agree with the ART results in Silva's work [48], which are obtained by using the trigonometric functions to express the incident potential in the far field. The general expressions in Eq. (10) demonstrate the possibility of calculation of the three-dimensional ARTs of objects in arbitrary fields once the incident and scattered beam shape coefficients can be computed properly, and in fact, could be applied for arbitrary acoustic waves once the incident beam shape coefficients are available.

\section{B. Beam shape coefficients of a Bessel beam with arbitrary location}

Bessel beams are one kind of typical vortex beams, which are a theoretical solution to the scalar Helmholtz equation. The essential point to describe a vortex beam in theoretical and numerical methods is to obtain the incident beam shape coefficients $a_{n m}$ on a given basis function. In this paper, Bessel beams with arbitrary topological charges (or orders) $M$ and locations $O_{\mathrm{VB}}$ relative to the particle center (as shown in Fig. 1) are considered with the acoustic potential

$$
\Phi_{B}=\Phi_{0} i^{M} e^{i k_{z}\left(z-z_{0}\right)} J_{M}\left(k_{r} R^{\prime}\right) e^{i M \varphi^{\prime}},
$$

where $k_{r}=k \sin \beta$ and $k_{z}=k \cos \beta$ depict the radial and axial components of the wave number with $\beta$ the cone angle of the Bessel beam, $R^{\prime}=\sqrt{\left(x-x_{0}\right)^{2}+\left(y-y_{0}\right)^{2}}$ gives the radial distance of a field point $(x, y, z)$ to the beam origin $O_{\mathrm{VB}},\left(x_{0}, y_{0}, z_{0}\right)$, and $\varphi^{\prime}=\tan ^{-1}\left[\left(y-y_{0}\right) /\left(x-x_{0}\right)\right]$ is the relative azimuthal angle. Note that Eq. (11) degrades into the special cases of on-axis incidence situation $[3,4,44]$ when the particle center is located on the axis of Bessel beams. By using the Graf's addition theorem of Bessel functions, the incident beam shape coefficients $a_{n m}$ were first derived theoretically in our previous work [33]

$$
a_{n m}=4 \pi \xi_{n m} i^{n-m+M} P_{n}^{m}(\cos \beta) J_{m-M}\left(\sigma_{0}\right) e^{-i k_{z} z_{0}} e^{-i(m-M) \varphi_{0}},
$$

where $\xi_{n m}=[(2 n+1)(n-m) !]^{1 / 2}[4 \pi(n+m) !]^{-1 / 2}$ are the normalized coefficients, $P_{n}^{m}$ are the associated Legendre functions, $\sigma_{0}=k_{r} R_{0}, R_{0}=\sqrt{x_{0}^{2}+y_{0}^{2}}$, and $\varphi_{0}=$ $\tan ^{-1}\left(y_{0} / x_{0}\right)$.

In order to obtain the three-dimensional ARTs of the particle in an ideal fluid according to Eq. (10), the scattered (beam shape) coefficients need to be calculated with a suitable method. In the present work, both the theoretical method (PWSM) for spherical shapes and the semi-analytical and semi-numerical method (TMM) for nonspherical shapes are introduced to study the rotational characteristics of the particles of interest. Both the PWSM and TMM give a linear relationship between the incident $\left(a_{n m}\right)$ and scattered $\left(s_{n m}\right)$ beam shape coefficients. For the PWSM, the relation could be described as $s_{n m}=$ $A_{n} a_{n m}$, where the partial wave coefficients $A_{n}$ are related to the complex functions (scattering coefficients) $s_{n}: A_{n}=$ $\left(s_{n}-1\right) / 2$. The scattering coefficients $s_{n}$ are known for a wide variety of spheres [44] and could be easily extended for spherical shapes with different material compositions. On the other hand, the transition relationship between $a_{n m}$ 
and $s_{n m}$ in TMM is described by $s_{n m}=T_{n m, n^{\prime} m^{\prime}} a_{n^{\prime} m^{\prime}}$, where $T_{n m, n^{\prime} m^{\prime}}$ denotes the transition matrix, which only depends on the properties of the object, including the geometrical shape, the material composition, and the boundary conditions at the interface, and otherwise is independent of the sources. For the PWSM, the partial wave coefficients $A_{n}$ could be regarded as the transition matrix $T_{n n^{\prime}}=$ $\left(s_{n}-1\right) / 2$ without the dependence on the azimuthal index $m$ for spheres. The TMM has been verified for the scattering of several typical rigid nonspherical objects [39-43] and hence could be extended for ART computations in the present work. In the following computations, the truncation number is set as $N_{\max }=2+\operatorname{Int}\left(8+k r_{0}+4.05 \sqrt[3]{k r_{0}}\right)$ (Int denotes the integer part of the indicated argument), which could ensure the accuracy and convergence of the present computations of PWSM and TMM [38].

\section{THEORETICAL AND NUMERICAL RESULTS}

\section{A. Validation and resonance scattering explanation with PWSM}

The transfer of angular momentum could come from dissipation mechanisms by the absorption either in the absorbing particle or the viscous boundary layer in the surrounding fluid [37]. In this work, an ideal fluid without the viscosity and only the spinning motions around the particle centroid are considered to investigate the threedimensional ARTs of spherical and nonspherical shapes in a single Bessel beam. To verify the theory and homemade programming, a viscoelastic solid sphere located on the axis of the first-order Bessel beam is studied. The sphere is made of polyethylene (PE) [49] with density $\rho_{e}=$ $957 \mathrm{~kg} / \mathrm{m}^{3}$, and longitudinal and transverse sound speeds $c_{p}=2430 \mathrm{~m} / \mathrm{s}, c_{s}=950 \mathrm{~m} / \mathrm{s}$, respectively. The normalized absorption coefficients are $\gamma_{p}=0.0074$ and $\gamma_{s}=$ 0.022 , respectively. The surrounding fluid is an ideal water with density $\rho_{0}=1000 \mathrm{~kg} / \mathrm{m}^{3}$ and speed of sound $c_{0}=$ $1500 \mathrm{~m} / \mathrm{s}$. As shown in Fig. 2, the characteristic length of the spherical shell is the outer radius $r_{0}=a$ and the inner radius is $b . b=0$ gives the special case for a solid sphere and the shell case will be discussed in Sec. B. The partial wave coefficients $A_{n}$ for the viscoelastic spherical shell could be obtained by using the complex wave numbers instead of those in elastic materials [43,50]. When the spherical shell case turns into a solid sphere, Eq. (18) in Ref. [50] [Eq. (A2) in Appendix A] should choose the first and second rows with the first and third columns both for the numerator and denominator according to the boundary condition and continuity [43], as shown in the Appendix A.

The dimensionless axial ARTs of the solid PE sphere are depicted versus the dimensionless frequency $k a$ and cone angle $\beta$ of the first-order $(M=1)$ Bessel beam for the on-axis incidence [transverse offset $\left(x_{0}, y_{0}\right)=(0,0)$ ], as shown in Fig. 2. The present theory could be applied for

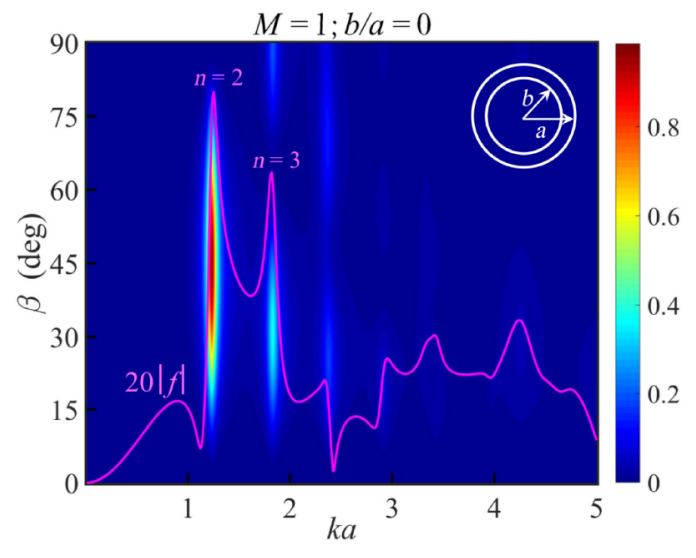

FIG. 2. The two-dimensional axial acoustic radiation toque pattern of a viscoelastic solid sphere $(b / a=0)$ vs $(k a, \beta)$ with $k a$ the dimensionless frequency and $\beta$ the cone angle of the first-kind Bessel beam (order $M=1$ ). The modulus of the dimensionless resonance backscattering form function (see text) from the solid sphere by an ordinary plane wave is scaled by a ratio of 20 with the magenta solid line. The frequencies of the quadrupole $(n=2)$ and octupole $(n=3)$ scattering are marked out where the axial ART peaks occur.

both the on-axis and off-axis incidences of a Bessel beam illuminating a particle. In other words, when the origin of the beam coincides with the particle center, our theory could degrade into the special case of on-axis incidence, as derived previously [32]. The theory and homemade programming could be validated according to the theoretical expression $T_{z}=(M / \omega) P_{\text {abs }}$ and could be extended for more general situations. To explain the physical mechanism for the maximum axial ARTs, the modulus of dimensionless resonance backscattering form function (given in the Appendix A) in an ordinary plane wave is also depicted in Fig. 2 with a scale ratio of 20 (the magenta solid line), which is obtained by subtracting a complex rigid background according to the resonance scattering theory [51]. As observed, the frequencies of ART peaks appear beyond the Rayleigh regime and coincide with those of resonance scattering peaks (especially the quadrupole $(n=2)$ and octupole $(n=3)$ scattering, respectively). The physical interpretation may be given as: based on the energy conservation, the vibration modes at the resonance frequencies will absorb more energy (i.e., larger $P_{\text {abs }}$ ) from the Bessel beam with the same topological charge $(M)$ and angular frequency $(\omega)$, resulting in a larger transfer of orbital angular momentum and accordingly larger axial ARTs, as illustrated by $T_{z}=(M / \omega) P_{\text {abs }}$. This result has also been studied recently by Baresch et al., who concluded that the dipolar and quadrupolar particle vibration modes dominate for the ART even in the Rayleigh scattering regime [37]. Furthermore, as shown in Fig. 2, there is no negative axial ARTs (i.e., ART reversal), which is easily understood from $T_{z}=(M / \omega) P_{\text {abs }}>0$ since $M>0, \omega>0$, and 
$P_{\text {abs }}>0$ for the considered situation. However, the theory will fail when the particle center moves off the beam axis. The axial ART may reverse under off-axis incidence and will be further investigated in the following.

\section{B. Reversal of axial radiation torque and physical mechanism with PWSM}

The PWSM is employed to study the axial ARTs for the PE spherical shell and solid sphere. There is air inside the shell with density $\rho_{i}=1.23 \mathrm{~kg} / \mathrm{m}^{3}$ and sound speed $c_{i}=$ $340 \mathrm{~m} / \mathrm{s}$. The reversal of axial ARTs for a polyurethane sphere in a first-order Bessel beam has been observed [48], however, the physical mechanism and parametric conditions have not yet revealed, which is one of the motivations of the present work. As shown in Fig. 3, the two-dimensional axial ARTs patterns versus $(k a, \beta)$ for a spherical shell (the first row, aspect ratio $b / a=0.99$ ) and solid sphere (the second and third rows, $b / a=0$ ) in a Bessel vortex beam are presented with different offsets: $\left(x_{0}, y_{0}\right)=(\pi / k a, \pi / k a),(2 \pi / k a, 2 \pi / k a),(3 \pi / k a, 3 \pi / k a)$ for the first to third columns with the distances in the unit of meters. Obviously, the reversal ARTs could be observed at certain dimensionless frequencies and cone angles. Note that the axial ART of a sphere will vanish for the zeroth-order Bessel beam even in the off-axis incidence since there is no orbital angular momentum in the ordinary Bessel beam [31], which is not given here. However, the axial ART may still exist for nonspherical shapes with the off-axis situation. Similar to the results in Fig. 2, the maximum modulus of axial ARTs lies at the frequencies of resonance scattering due to the large absorption energy. In addition, when the particle deviates further away from the beam axis (with larger offset), the high-order scattering (a)

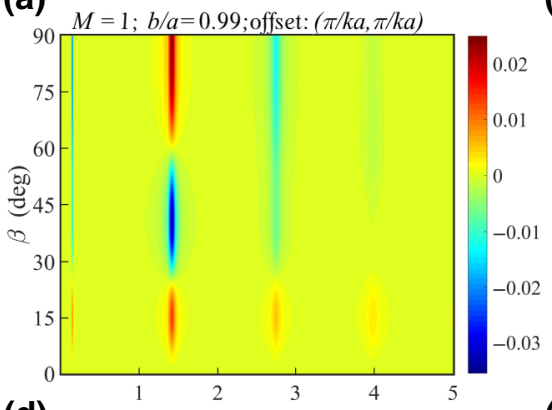

(d)

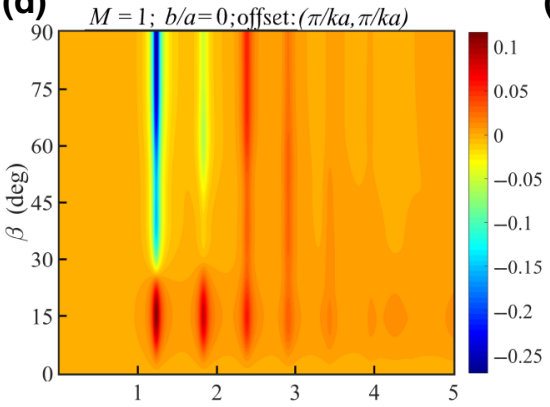

(g)

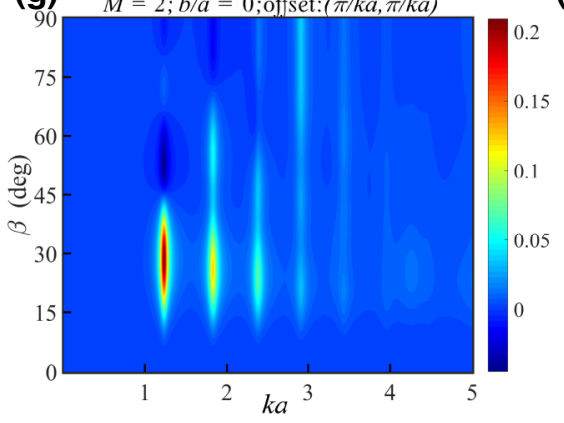

(b)

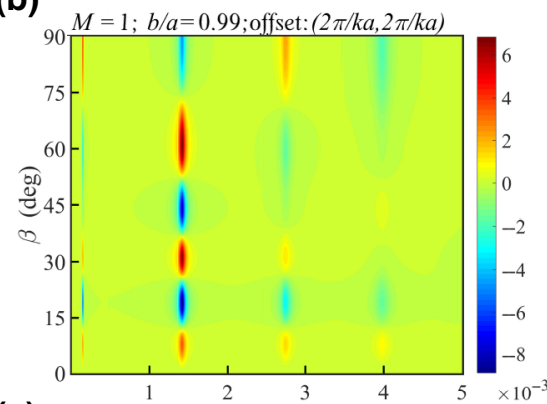

(e)

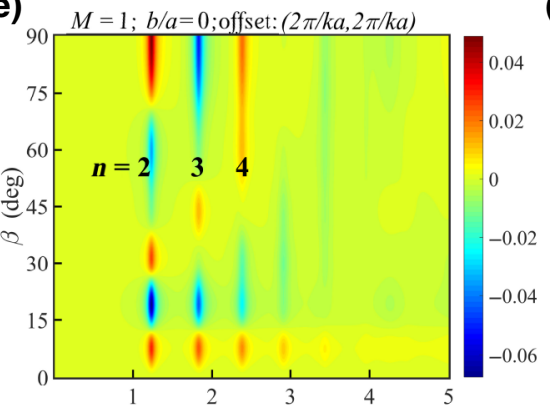

(h)

h) $M=2 ; b / a=0 ;$ offset: $(2 \pi / k a, 2 \pi / k a)$

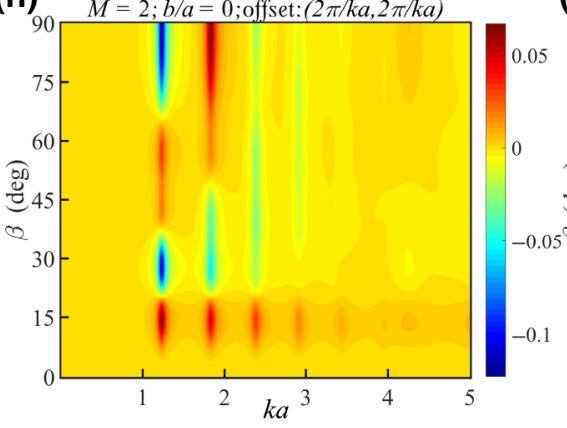

(c)

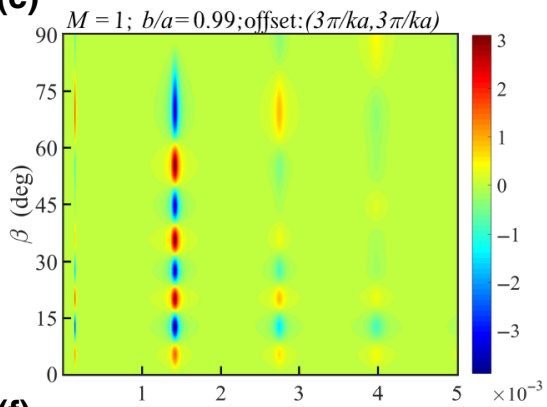

(f)

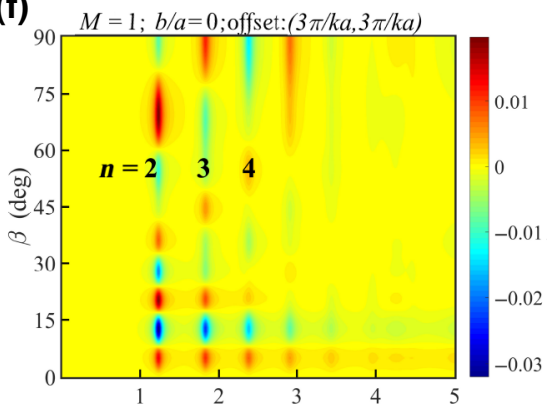

(i)

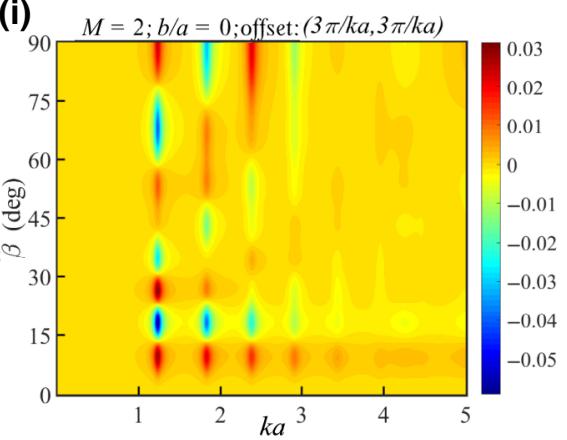

FIG. 3. The two-dimensional axial acoustic radiation toque patterns for $(\mathrm{a}-\mathrm{c})$ spherical shell with aspect ratio $b / a=0.99$ and $(\mathrm{d}-\mathrm{i})$ solid sphere with $b / a=0$. The first-order for $(\mathrm{a}-\mathrm{f})$ and second-order for $(\mathrm{g}-\mathrm{i})$ Bessel beams are considered with off-axis incidence (The offset is in the length unit of meter.): $\left(x_{0}, y_{0}\right)=(\pi / k a, \pi / k a)$ for the first, $\left(x_{0}, y_{0}\right)=(2 \pi / k a, 2 \pi / k a)$ for the second, and $\left(x_{0}, y_{0}\right)=$ $(3 \pi / k a, 3 \pi / k a)$ for the third column, respectively. The numbers of scattering modes $(n=2,3,4)$ are also marked out in panels $(\mathrm{e})$ and (f) at frequencies where the axial ART reverses. 


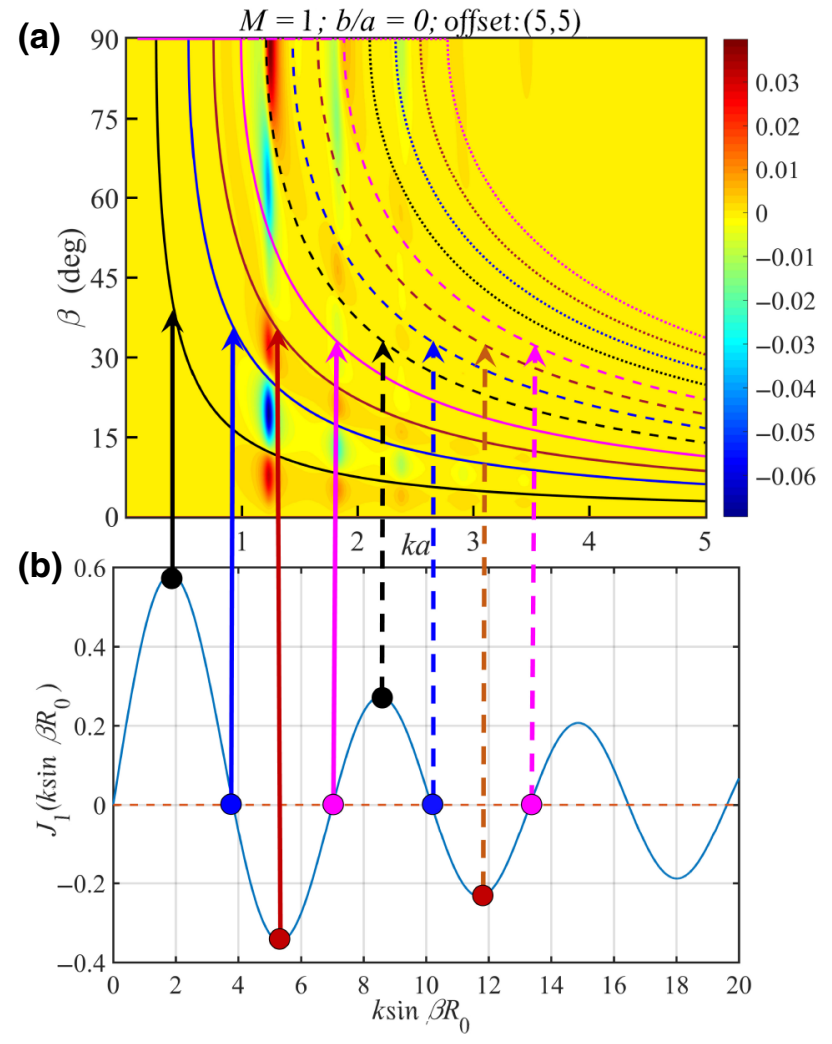

FIG. 4. (a) The two-dimensional axial ARTs pattern of a PE solid sphere $(b / a=0)$ illuminated by the first-order $(M=1)$ Bessel beam with a fixed offset, $\left(x_{0}, y_{0}\right)=(5,5)$. (b) The cylindrical Bessel function of the first kind versus $k_{r} R_{0}=k \sin \beta R_{0}$. The ART reversals occur on the $\beta-k a$ lines with the parameters of $\beta$ and $k a$, which makes $J_{1}\left(k \sin \beta R_{0}\right)$ the extremum or zero values.

modes, including the octupole $(n=3)$ and hexadecapole $(n=4)$ or even higher orders as marked in Figs. 3(e) and 3(f), also play a dominant role in the axial ART for the off-axis incidence situation. However, the maximum torque will decrease with the increase of offset. This phenomenon and principle may be very meaningful for acoustic rotation of elastic particles beyond the Rayleigh regime. It is interesting to note that the intervals of both the thin shell and solid sphere are almost equally spaced along the dimensionless frequency $k a$, due to the leak of elastic wave components, which circumnavigate the shell or sphere repeatedly (the zeroth-order symmetric and antisymmetric Lamb modes are for thin shells [52], while the Rayleigh surface wave is for solid spheres). Comparing the axial ARTs of the second-order case (third row for $M=2$ ) with the first-order (second row for $M=1$ ), higher topological charges tend to give a larger maximum torque since beams with larger topological charges carry more orbital angular momentum [32].

To further study the physical mechanism and the parameter conditions of the reversals, the axial ARTs of a PE sphere $(b / a=0)$ illuminated by the first-order $(M=1)$
Bessel beam with a fixed offset $\left(x_{0}, y_{0}\right)=(5,5)$ are depicted [Fig. 4(a)] as well as the cylindrical Bessel function of the first kind versus $k_{r} R_{0}=k \sin \beta R_{0}$ [Fig. 4(b)]. As shown in Eq. (11), the velocity potential of the Bessel beam depends on the cylindrical Bessel function $J_{M}\left(k_{r} R^{\prime}\right)$. It is more important to discuss the properties of $J_{M}\left(k_{r} R_{0}\right)$ rather than those of $J_{M}\left(k_{r} R^{\prime}\right)$, which is only related to the offset of the particle to the beam axis and independent of the arbitrary field point $(x, y, z)$. Similar to the results in Fig. 3, Fig. 4(a) also depicts the reversals of axial ARTs, especially at certain fixed $k a$ and different $\beta$. To find out the parameter conditions when the reversal appears, the $k a-\beta$ curves are also plotted in Fig. 4(a), which meets $k \sin \beta R_{0}=1.85,3.832,5.335,7.016$, $8.551,10.17$, and 11.71 [in fact the roots of the extremum or zero values of $J_{1}\left(k_{r} R_{0}\right)$ from left to right as shown in Fig. 4(b)]. It is obvious to observe that the reversals (the negative and positive ARTs exchange) will occur when the parameters of dimensionless frequency $k a$ and cone angle $\beta$ are located on the $k a-\beta$ curves in Fig. 4(a). This phenomenon has been found independently by numerical experiments for the parameter condition of axial ART reversal at broadband frequencies (beyond the Rayleigh limit) $[42,43]$, which coincides with the recent theoretical derivation for a small particle in the Rayleigh limit [i.e., $\left.J_{M}\left(k_{r} R_{0}\right) J_{M}^{\prime}\left(k_{r} R_{0}\right)=0\right]$ [31].

\section{Reversal of transverse radiation torque of rigid spheroid with TMM}

For the experimental setup, it may be challenging for the calibration of the vortex beam and the center of particles. Hence, one technology of the vortex-beam-based acoustic tweezers may be promising, which could reverse the torque without rotating the devices of the experimental transducers and only need to tune the cone angle or incident frequency. In the long-wave approximation, the interactions between the acoustic field and particle will not be sensitive to the particle shapes, while the geometrical shape effect may dominate beyond the Rayleigh scattering regime. To this end, the traditional TMM is introduced to study the ARTs combined with the derived formulas in Sec. II. Considering a rigid spheroid centered on the axis of the zeroth-order Bessel beam $(M=0)$, as shown in Fig. 5(a), the incident beam shape coefficients are derived using the addition theorem of spherical harmonics [39]

$a_{n m}=4 \pi \xi_{n m} i^{n} P_{n}^{m}\left(\cos \theta_{i}\right) P_{n}^{m}(\cos \beta)\left(\begin{array}{ll}\cos \left(m \varphi_{i}\right), & \sigma=e \\ \sin \left(m \varphi_{i}\right), & \sigma=o\end{array}\right)$,

where $\theta_{i}$ and $\varphi_{i}$ are the polar and azimuthal angles of the incident beam and $\sigma=e, o$ (even, odd) specifies the azimuthal parity, which is introduced to compute the transition matrix for convenience [41]. The transition matrix 

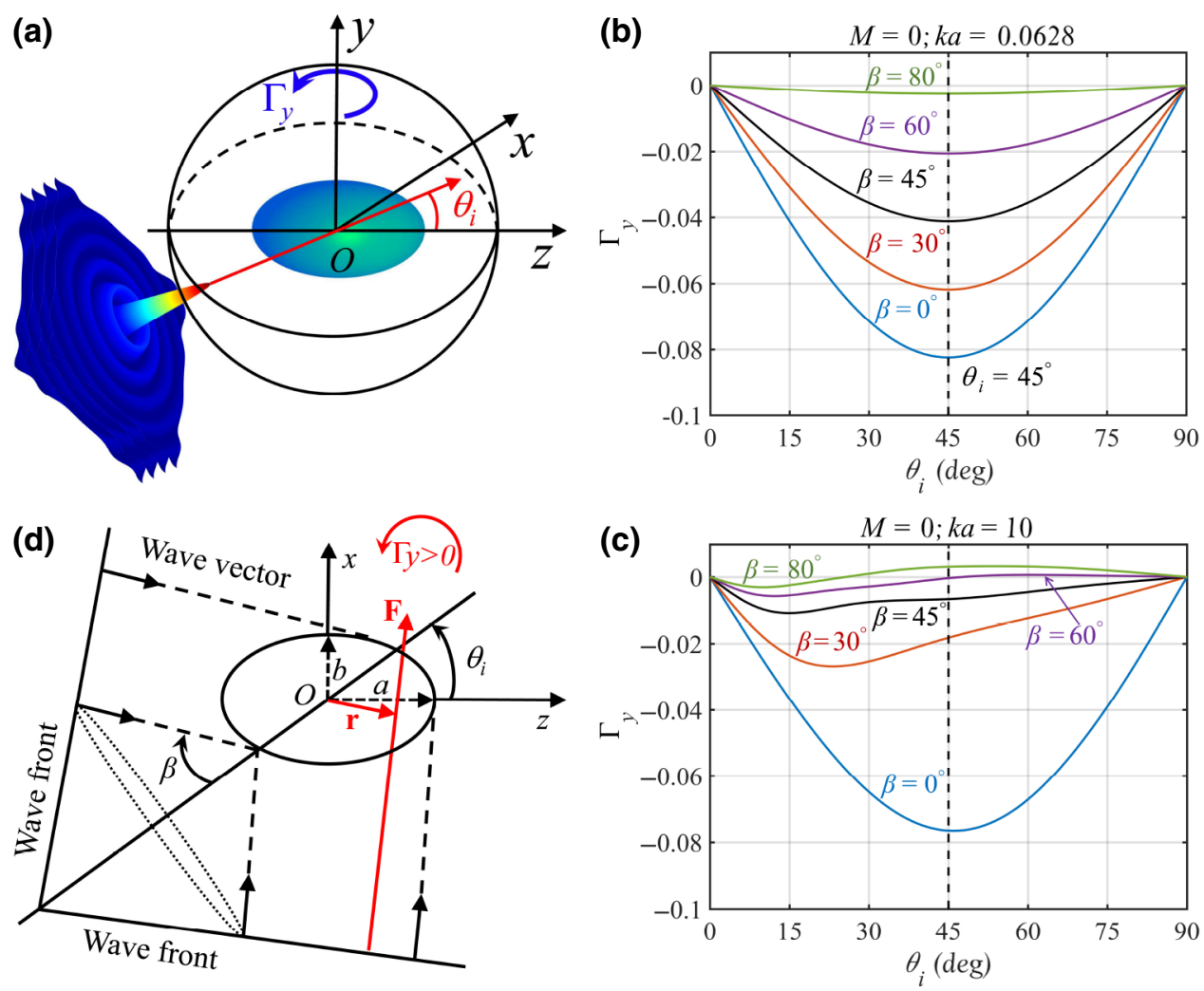

FIG. 5. (a) The schematic of a rigid spheroid (aspect ratio $b / a=0.5)$ centered on the axis of the zeroth-order Bessel beam $(M=0)$ with the incident polar angle $\theta_{i}$. (b, c) the transverse ARTs $\Gamma_{y}$ curves versus the incident angle $\theta_{i}$ in the Rayleigh $(k a=0.0628)$ and geometric-optics regimes $(k a=10)$. Different cone angles are selected with $\beta=0^{\circ}$ (plane wave case) and $\beta=30^{\circ}, 45^{\circ}$, $60^{\circ}$, and $80^{\circ}$. (d) The geometrical ray diagram of the zeroth-order Bessel beam with oblique incidence to qualitatively explain the reversal of the transverse ART $\Gamma_{y}$. could be calculated by Eqs. (14) and (15) from Ref. [39], which is given in Appendix B. When the axis of the beam coincides with that of the rotational axis of the spheroid, the axial ART will vanish for the symmetry and out of absorption. However, the transverse ARTs will be induced because of the asymmetrical scattering, which leads to the spinning motions.

A rigid spheroid with the aspect ratio $b / a=0.5$ (here, $r_{0}=a$ ) is illuminated by the ordinate Bessel beam with normal or oblique incidences (i.e., different $\theta_{i}$ ), where $a$ and $b$ are the polar and equatorial radii as shown in Fig. 5(d). The geometrical shape function of the spheroid is $r(\theta)=\left(\cos ^{2} \theta / a^{2}+\sin ^{2} \theta / b^{2}\right)^{-1 / 2}$ with $\theta$ the polar angle, which is independent of the azimuthal angle for the rotational symmetry. The transverse ART $\Gamma_{y}$ with different cone angles $\beta$ are investigated at frequencies lying in both the Rayleigh limit [Fig. 5(b): $k a=0.0628$ ] and geometricoptics regime [Fig. 5(c): $k a=10$ ]. $\beta=0^{\circ}$ gives the special case when the Bessel beam turns into an ordinary plane wave. The black dashed line describes $\theta_{i}=45^{\circ}$. As shown in Fig. 5(b) in the Rayleigh limit, the ART $\Gamma_{y}$ vanishes with normal $\theta_{i}=0^{\circ}$ or broadside $\theta_{i}=90^{\circ}$ incidence for symmetry and the maximum modulus occurs at $\theta_{i}=45^{\circ}$. The direction of the ART is defined based on the right-hand rule. This phenomenon agrees with the theoretical results of a rigid spheroid in a plane standing-wave field by Fan et al. with $\Gamma_{y}$ proportional to $\sin \left(2 \theta_{i}\right)$, suggesting the maximum modulus of ART occurs at $\theta_{i}=45^{\circ}$ [53]. Since in the Rayleigh regime the wavelength is much larger than the characteristic length $r_{0}=a$ of the spheroid, the transverse torque $\Gamma_{y}$ is not sensitive to the geometrical crosssection of the spheroid, leading to the symmetry about $\theta_{i}=45^{\circ}$ of the $\Gamma_{y} \sim \theta_{i}$ curves depicted in Fig. 5(b). However, the crosssection will play an important role when the particle size is comparable to the wavelength, for example, in the geometric-optics regime. The results are shown in Fig. 5 (c) and the symmetry about $\theta_{i}=45^{\circ}$ of the $\Gamma_{y} \sim \theta_{i}$ curve breaks as expected for the variety of the crosssection of the spheroid as the incident polar angle changes. In addition, the transverse ART reverses at certain cone angles when the incident polar angle $\theta_{i}$ is larger than a critical value. This could be explained with the acoustic rays diagram as shown in Fig. 5(d): the beam vectors deviate the axis with a cone angle $\beta$ for Bessel beams and the red-marked rays dominate to produce a larger positive $\Gamma_{y}$. It is easier to obtain the ART reversal for a larger cone angle (e.g., beam with $\beta=80^{\circ}$ reverses at $\theta_{i}=25^{\circ}$ while with $\beta=60^{\circ}$ it reverses at $\theta_{i}=48^{\circ}$ ). The present work could be extended for other kinds of geometrical shapes if the geometrical function is available, for example, the finite cylinder with endcaps $[40,41]$.

\section{Roughness effect on the transverse ARTs with TMM}

The surface roughness of particles will affect the acoustic scattering in three-dimensional space and thus alter the transfer of linear and angular momenta between the 

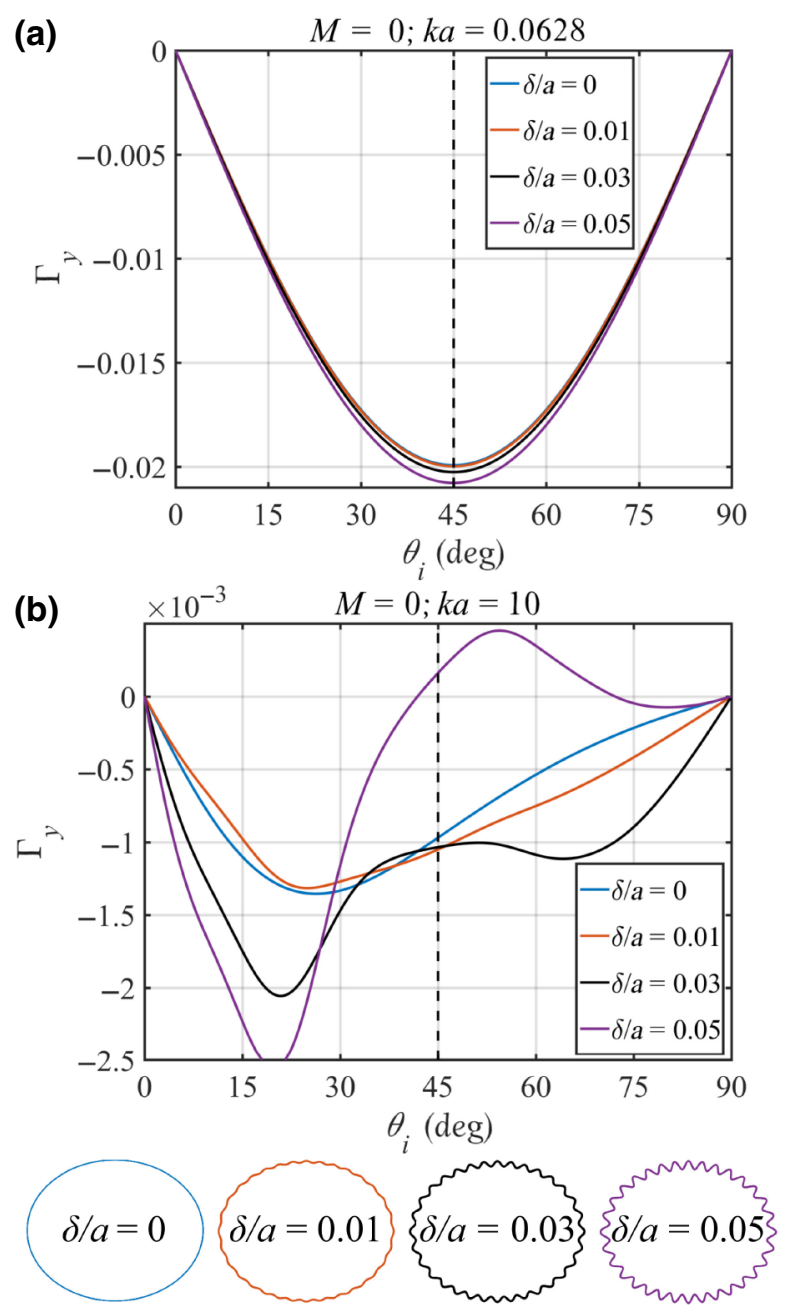

FIG. 6. Similar to Figs. 5(b) and 5(c) except that the aspect ratio of the rigid spheroid is $b / a=0.8$ with rough surfaces at dimensionless frequencies (a) $k a=0.0628$ and (b) $k a=10$, respectively. The rough spheroid is still rotationally axisymmetric. The two-dimensional rough spheroids are depicted at the bottom with the relative roughness $\delta / a=0$ (smooth spheroid), $0.01,0.03$, and 0.05 .

acoustic field and particles. This section will discuss the effect of surface roughness of a rigid nonspherical shape on the dimensional ARTs with the same expression of incident beam shape coefficients (zeroth-order Bessel beam with a fixed cone angle $\beta=60^{\circ}$ ). The geometrical shape function of the rough spheroid is $r(\theta)=$ $\left(\cos ^{2} \theta / a^{2}+\sin ^{2} \theta / b^{2}\right)^{-1 / 2}+\delta \cos (l \theta)$ with $\delta$ the amplitude of the roughness and $l$ the periodic number of the roughness around the spheroid (a multiple of four), as shown in Fig. 6 with aspect ratio $b / a=0.8$ (here, $\left.r_{0}=a\right), l=32, \delta / a=0$ (smooth spheroid), and $\delta / a=$ $0.01,0.03$, and 0.05 (rough spheroids). Note that the roughness amplitude $\delta$ could be random over the geometrical shape of the particle, which will be given as a statistical distribution. For simplicity, only the fixed surface roughness is taken into consideration in the present work. The convergence test is conducted for the rough spheroid with $k a=10, \delta / a=0.05$ and demonstrates the correctness of our homemade programming. The convergence is achieved at $N_{\max }=19$, which is smaller than the truncation number applied in the present work having $N_{\max }=$ $2+\operatorname{Int}\left(8+k r_{0}+4.05 \sqrt[3]{k r_{0}}\right)=29$. As is similar to the case in Sec. III C with the rough spheroid replacing the smooth one, the dimensionless ARTs around the $y$ axis of the rough spheroids with different roughnesses under the illumination of the zeroth-order Bessel beam are described versus the incident polar angle $\theta_{i}$ at dimensionless frequencies $k a=0.0628$ and $k a=10$, respectively. Once again, as shown in Fig. 6(a), the symmetries of the $\Gamma_{y} \sim \theta_{i}$ curves still exist in the Rayleigh regime $(k a=0.0628$ with a wavelength much larger than the particle size and roughness $\delta$ ). This is because the geometrical shapes of the rough spheroids are still symmetric. However, a slight increase of the roughness will lead to a small increase in the ART, probably due to the interactions between the subwavelength structure along the geometrical surface of the rough spheroid and the acoustic field. However, the physical mechanism is outside the scope of the present work. For the effect of the roughness on the transverse ART in the geometric-optics regime $(k a=10)$, the roughness plays a dominant role to tune the $\Gamma_{y}$ versus the polar angle $\theta_{i}$, as shown in Fig. 6(b). This phenomenon suggests that the surface shape with geometrical roughness may not be neglected to model several biological cells or bacterial at relatively high frequencies. At the broadband frequencies, the more accurate model could provide a better forecast of the three-dimensional ART as well as acoustic radiation forces to better control particles or cells in acoustics.

\section{DISCUSSION AND CONCLUSION}

The spinning rotation of a particle around its own axes in an acoustic wave field describes extra degrees of freedom besides the translocations and orbital rotations for acoustic tweezers. The nonviscous fluid is considered in this work and hence the radiation angular momentum stress tensor theory $[45,46]$ could be applied to compute the ARTs by transferring the integrals over the geometrical shape of the particle to a standard spherical surface in the far field. In addition, theoretical formulas are derived independently and proven to express the three-dimensional ARTs in terms of the incident and scattered beam shape coefficients, which could be calculated with the PWSM [3-5] and TMM [33,39-43]. Note that the dissipations both in the bulk of an absorbing particle and in the viscous boundary layer in the surrounding fluid play a dominant role to induce the ARTs [37]. In addition, the spinning rate $\Omega$ for a Rayleigh particle in a Bessel beam could be evaluated by keeping the balance between the axial ART and the 
viscous-drag torque $T_{D}=8 \pi a^{3} v \rho_{0} \Omega$, where $a$ is the particle size, $v$ is the kinematic viscosity, and $\rho_{0}$ is the fluid density outside [31,37]. Viscous effects on the acoustic torque neglected here can be unimportant when the oscillating viscous boundary layer is small in comparison with the object size, the effects of streaming are negligible, and dissipation mechanisms within the particle are dominant. Our approach is similar to that in Ref. [32], which predicted torques on an absorbing disk in an acoustic vortex beam in agreement with measurements [54]. An approximation for estimating viscous contributions to the acoustic torque for small spheres given in Ref. [55] is supported by numerical simulation in a relevant situation [56].

Both the axial and transverse ARTs are investigated with emphasis on the parameters' conditions and corresponding physical mechanisms of the radiation torque reversals. The spherical and nonspherical particles are studied by using the partial wave series method and the T matrix method, respectively. It is found independently that the axial torque will reverse at certain parameter conditions verified by the combination of numerical computations and theoretical formulas, which indicates that the dipole, quadrupole, and even higher-order scattering modes have a large contribution to the radiation torque when the absorbing particle is placed off the beam axis. Note that even in the Rayleigh limit, the quadrupole vibration mode in the particle dominates to induce the axial torque, and hence the long-wave approximation will fail to obtain the total ARTs [37]. Meanwhile, higher-order vibration modes (e.g., octupole with $n=3$ and hexadecapole with $n=4$ ) play a dominant role for the ARTs when the particle moves far away from the beam axis (with larger offsets). The resonance scattering seems to lead to larger energy absorption from the acoustic field by the energy conservation, resulting in the larger ARTs at the resonance frequencies.

For the rigid spheroid, the transverse ART shows the symmetry on the incidence angles in the Rayleigh regime for both the smooth and rough geometrical surfaces since the particle size and roughness is much smaller than the long wavelength, leading to the fact the induced ARTs are not sensitive to the geometrical shapes of the particles. On the contrary, the transverse ARTs will depend on the shapes in the geometric-optics regime and the symmetry on the incident angle is hence broken for the different crosssections of the spheroid when the incident direction varies. Furthermore, the transverse ART will reverse with a relatively larger cone angle of the Bessel beam under the oblique incidence and this can be understood qualitatively by a geometrical rays diagram. The roughness on the geometrical shape is also discussed and found to be important since it affects the ARTs in the geometricoptics regime and can be neglected in the long-wavelength approximation.

In the fields of life sciences [25] and microfluidics, acoustic tweezers are playing a more and more important role for precise control of particles in acoustics, which mainly consider the translocation and orbital rotation induced by the acoustic radiation forces or the drag force from acoustic streaming, and the spinning rotation induced by the acoustic radiation torques. Hence, it is helpful to consider more degrees of freedom at broadband frequencies (including the Rayleigh limit and Mie and geometricoptics regimes) and more precise geometrical models, leading to the applicability to explore new physical mechanisms and applications in biomedical science and lab-on-achip technology. The present work provides improvements to some extent on these alternative phenomenon and physical mechanisms for the ART reversal in the spinning motion dynamics, which may be beneficial to experimental designs and engineering applications.

\section{ACKNOWLEDGMENTS}

Work supported by HUST Postgraduate Overseas Shortterm Study Program Scholarship and China Scholarship Council (Z.G.), NSFC (W.L. and Z.G.), and ONR (P.L.M). The authors are grateful for helpful discussions with Dr. Likun Zhang at the University of Mississippi.

\section{APPENDIX A: PARTIAL WAVE COEFFICIENTS FOR VISCOELASTIC SPHERICAL SHELL FILLED WITH FLUID}

For convenience, several dimensionless frequencies are introduced as [43]: $x=k a, x_{p}=k_{p} a, x_{s}=k_{s} a, z_{p}=k_{p} b$, $z_{s}=k_{s} b$, and $z_{i}=k_{i} b$, where $a$ and $b$ describe the outer and inner radii of the spherical shell and $k, k_{p}, k_{s}$, and $k_{i}$ are the wave numbers in the outer fluid, in the elastic shell of longitudinal and shear components, and in the inner fluid. In the outer fluid, the velocity and density are $\rho_{0}$ and $c_{0}$. In the elastic shell, the density is $\rho_{e}$ and the longitudinal and shear velocities are $c_{p}$ and $c_{s}$. In the inner fluid, the velocity and density are $\rho_{i}$ and $c_{i}$. The partial wave coefficients $A_{n}$ (instead of scattering coefficients $s_{n}$ ) are given as [50]

$$
A_{n}=-\frac{F_{n} j_{n}(x)-x j_{n}^{\prime}(x)}{F_{n} h_{n}^{(2)}(x)-x h_{n}^{(2)^{\prime}}(x)},
$$

where the coefficients $F_{n}$

$$
\begin{gathered}
F_{n}=-\rho_{0}\left|\begin{array}{ccccc}
\alpha_{22} & \alpha_{23} & \alpha_{24} & \alpha_{25} & 0 \\
\alpha_{32} & \alpha_{33} & \alpha_{34} & \alpha_{35} & 0 \\
\alpha_{42} & \alpha_{43} & \alpha_{44} & \alpha_{45} & \alpha_{46} \\
\alpha_{52} & \alpha_{53} & \alpha_{54} & \alpha_{55} & \alpha_{56} \\
\alpha_{62} & \alpha_{63} & \alpha_{64} & \alpha_{65} & 0
\end{array}\right| / \\
\left|\begin{array}{ccccc}
\alpha_{12} & \alpha_{13} & \alpha_{14} & \alpha_{15} & 0 \\
\alpha_{32} & \alpha_{33} & \alpha_{34} & \alpha_{35} & 0 \\
\alpha_{42} & \alpha_{43} & \alpha_{44} & \alpha_{45} & \alpha_{46} \\
\alpha_{52} & \alpha_{53} & \alpha_{54} & \alpha_{55} & \alpha_{56} \\
\alpha_{62} & \alpha_{63} & \alpha_{64} & \alpha_{65} & 0
\end{array}\right|
\end{gathered}
$$


with the matrix elements

$$
\begin{aligned}
& \alpha_{12}=\left(2 \rho_{e} / x_{s}^{2}\right)\left[\left(x_{p}^{2}-1 / 2 x_{s}^{2}\right) j_{n}\left(x_{p}\right)+x_{p}^{2} j_{n}^{\prime \prime}\left(x_{p}\right)\right], \\
& \alpha_{13}=\left(2 \rho_{e} / x_{s}^{2}\right)\left[\left(x_{p}^{2}-1 / 2 x_{s}^{2}\right) y_{n}\left(x_{p}\right)+x_{p}^{2} y_{n}^{\prime \prime}\left(x_{p}\right)\right] \text {, } \\
& \alpha_{14}=\left(2 \rho_{e} / x_{s}^{2}\right) n(n+1)\left[x_{s} j_{n}^{\prime}\left(x_{s}\right)-j_{n}\left(x_{s}\right)\right], \\
& \alpha_{15}=\left(2 \rho_{e} / x_{s}^{2}\right) n(n+1)\left[x_{s} y_{n}^{\prime}\left(x_{s}\right)-y_{n}\left(x_{s}\right)\right] \text {, } \\
& \alpha_{22}=x_{p} j_{n}^{\prime}\left(x_{p}\right) \text {, } \\
& \alpha_{23}=x_{p} y_{n}^{\prime}\left(x_{p}\right) \text {, } \\
& \alpha_{24}=n(n+1) j_{n}\left(x_{s}\right) \text {, } \\
& \alpha_{25}=n(n+1) y_{n}\left(x_{s}\right) \text {, } \\
& \alpha_{32}=2\left[x_{p} j_{n}^{\prime}\left(x_{p}\right)-j_{n}\left(x_{p}\right)\right] \text {, } \\
& \alpha_{33}=2\left[x_{p} y_{n}^{\prime}\left(x_{p}\right)-y_{n}\left(x_{p}\right)\right] \text {, } \\
& \alpha_{34}=\left(n^{2}+n-2\right) j_{n}\left(x_{s}\right)+x_{s}^{2} j_{n}^{\prime \prime}\left(x_{s}\right), \\
& \alpha_{35}=\left(n^{2}+n-2\right) y_{n}\left(x_{s}\right)+x_{s}^{2} y_{n}^{\prime \prime}\left(x_{s}\right) \text {, } \\
& \alpha_{42}=\left(2 \rho_{e} / z_{s}^{2}\right)\left[\left(z_{p}^{2}-1 / 2 z_{s}^{2}\right) j_{n}\left(z_{p}\right)+z_{p}^{2} j_{n}^{\prime \prime}\left(z_{p}\right)\right] \text {, } \\
& \alpha_{43}=\left(2 \rho_{e} / z_{s}^{2}\right)\left[\left(z_{p}^{2}-1 / 2 z_{s}^{2}\right) y_{n}\left(z_{p}\right)+z_{p}^{2} y_{n}^{\prime \prime}\left(z_{p}\right)\right] \text {, } \\
& \alpha_{44}=\left(2 \rho_{e} / z_{s}^{2}\right) n(n+1)\left[z_{s} j_{n}^{\prime}\left(z_{s}\right)-j_{n}\left(z_{s}\right)\right] \text {, } \\
& \alpha_{45}=\left(2 \rho_{e} / z_{s}^{2}\right) n(n+1)\left[z_{s} y_{n}^{\prime}\left(z_{s}\right)-y_{n}\left(z_{s}\right)\right] \text {, } \\
& \alpha_{46}=\rho_{i} j_{n}\left(z_{i}\right) \text {, } \\
& \alpha_{52}=z_{p} j_{n}^{\prime}\left(z_{p}\right) \text {, } \\
& \alpha_{53}=z_{p} y_{n}^{\prime}\left(z_{p}\right) \text {, } \\
& \alpha_{54}=n(n+1) j_{n}\left(z_{s}\right) \text {, } \\
& \alpha_{55}=n(n+1) y_{n}\left(z_{s}\right) \text {, } \\
& \alpha_{56}=-z_{j} j_{n}^{\prime}\left(z_{i}\right) \text {, } \\
& \alpha_{62}=2\left[z_{p} j_{n}^{\prime}\left(z_{p}\right)-j_{n}\left(z_{p}\right)\right], \\
& \alpha_{63}=2\left[z_{p} y_{n}^{\prime}\left(z_{p}\right)-y_{n}\left(z_{p}\right)\right] \text {, } \\
& \alpha_{64}=\left(n^{2}+n-2\right) j_{n}\left(z_{s}\right)+z_{s}^{2} j_{n}^{\prime \prime}\left(z_{s}\right) \text {, } \\
& \alpha_{65}=\left(n^{2}+n-2\right) y_{n}\left(z_{s}\right)+z_{s}^{2} y_{n}^{\prime \prime}\left(z_{s}\right),
\end{aligned}
$$

where $j_{n}$ and $y_{n}$ are the first and second kind of spherical Bessel functions, one (') and two (II) primes of functions represent the first- and second-order derivate with respect to the indicated argument. For the viscoelastic materials, the complex wave number should be introduced with $k_{p}^{*}=k_{p}\left(1-i \gamma_{p}\right)$ and $k_{s}^{*}=k_{s}\left(1-i \gamma_{s}\right)$ in replacement of the longitudinal and shear wave numbers in $x_{p, s}$ and $z_{p, s}$ where $\gamma_{p, s}$ are the normalized absorption coefficients, respectively. When the inner fluid is absent, the special case goes to the spherical solid situation. According to the linear equations of the boundary conditions, one has

$$
F_{n}=-\rho_{0}\left|\begin{array}{ll}
\alpha_{22} & \alpha_{24} \\
\alpha_{32} & \alpha_{34}
\end{array}\right| /\left|\begin{array}{ll}
\alpha_{12} & \alpha_{14} \\
\alpha_{32} & \alpha_{34}
\end{array}\right|
$$

Note that for a vacuum-filled spherical shell, the scattering coefficients are given in Refs. [57,58]. The far-field form function is defined in the PWSM as

$$
f(k a, \theta, \beta, \varphi)=\frac{-2 i}{k a} \sum_{n=0}^{N_{\max }} \sum_{m=-n}^{n} a_{n m} A_{n} Y_{n m}(\theta, \varphi) .
$$

For the nonspherical shape using the TMM, the outer radius of the spherical shape $a$ in Eq. (A5) should be replaced with the characteristic length $r_{0}$, and the partial wave coefficients $A_{n}$ should be replaced by the transition matrix.

\section{APPENDIX B: TRANSITION MATRIX FOR A RIGID ROTATIONALLY SYMMETRIC SHAPE}

As discussed in Sec. II B, the scattering coefficients could be obtained using the transition (T) matrix multiplying by the incident beam shape coefficients. For a rigid particle with rotational symmetry, the $\mathbf{T}$ matrix could be calculated as $\mathbf{T}=-\operatorname{Re} \mathbf{Q} \mathbf{Q}^{-1}$ with the element of the $\mathbf{Q}$ matrix [39]

$$
\begin{aligned}
\mathbf{Q}_{n m, n^{\prime} m^{\prime}}^{\sigma \sigma^{\prime}}= & \int_{0}^{\pi} \xi_{n^{\prime} m^{\prime} j_{n^{\prime}}}(k r) P_{n^{\prime}}^{m^{\prime}}(\cos \theta) \xi_{n m} \\
& \times\left[\frac{\partial h_{n}^{(1)}(k r)}{\partial r} P_{n}^{m}(\cos \theta)-\frac{r_{\theta}}{r^{2}} h_{n}^{(1)}(k r) \frac{\partial P_{n}^{m}(\cos \theta)}{\partial \theta}\right] \\
& \times r^{2} \sin \theta d \theta \int_{0}^{2 \pi}\left(\begin{array}{c}
\cos m^{\prime} \varphi \\
\sin m^{\prime} \varphi
\end{array}\right)\left(\begin{array}{c}
\cos m \varphi \\
\sin m \varphi
\end{array}\right) d \varphi,
\end{aligned}
$$

where $r(\theta)$ is the geometrical shape function and $r_{\theta}=$ $d r / d \theta$ is the derivate of $r(\theta)$ with respect to the polar angle $\theta$ on the particle surface. Further details and simplified methods can be found in Refs. [40,41].

[1] J. L. Thomas, R. Marchiano, and D. Baresch, Acoustical and optical radiation pressure and the development of single beam acoustical tweezers, J. Quant. Spectrosc. Radiat. Transf. 195, 55 (2017).

[2] D. Baresch, J. L. Thomas, and R. Marchiano, Observation of a Single-Beam Gradient Force Acoustical Trap for Elastic Particles: Acoustical Tweezers, Phys. Rev. Lett. 116, 024301 (2016).

[3] P. L. Marston, Axial radiation force of a Bessel beam on a sphere and direction reversal of the force, J. Acoust. Soc. Am. 120, 3518 (2006).

[4] P. L. Marston, Radiation force of a helicoidal Bessel beam on a sphere, J. Acoust. Soc. Am. 125, 3539 (2009).

[5] L. Zhang and P. L. Marston, Geometrical interpretation of negative radiation forces of acoustical Bessel beams on spheres, Phys. Rev. E 84, 035601 (2011).

[6] G. T. Silva, An expression for the radiation force exerted by an acoustic beam with arbitrary wavefront (L), J. Acoust. Soc. Am. 130, 3541 (2011). 
[7] S. Xu, C. Qiu, and Z. Liu, Transversally stable acoustic pulling force produced by two crossed plane waves, Europhys. Lett. 99, 44003 (2012).

[8] H. Bruus, Acoustofluidics 7: The acoustic radiation force on small particles, Lab Chip 12, 1014 (2012).

[9] D. Baresch, J. L. Thomas, and R. Marchiano, Threedimensional acoustic radiation force on an arbitrarily located elastic sphere, J. Acoust. Soc. Am. 133, 25 (2013).

[10] O. A. Sapozhnikov and M. R. Bailey, Radiation force of an arbitrary acoustic beam on an elastic sphere in a fluid, J. Acoust. Soc. Am. 133, 661 (2013).

[11] Z. Gong, P. L. Marston, and W. Li, T-matrix evaluation of acoustic radiation forces on nonspherical objects in Bessel beams, arXiv:1710.00146v1 (2017).

[12] M. F. Hamilton, Y. A. Ilinskii, and E. A. Zabolotskaya, Acoustic streaming generated by standing waves in twodimensional channels of arbitrary width, J. Acoust. Soc. Am. 113, 153 (2003).

[13] S. S. Sadhal, Acoustofluidics 13: Analysis of acoustic streaming by perturbation methods, Lab Chip 12, 2292 (2012).

[14] R. Barnkob, P. Augustsson, T. Laurell, and H. Bruus, Acoustic radiation- and streaming-induced microparticle velocities determined by microparticle image velocimetry in an ultrasound symmetry plane, Phys. Rev. E 86, 056307 (2012).

[15] F. Guo, Z. Mao, Y. Chen, Z. Xie, J. P. Lata, P. Li, L. Ren, J. Liu, J. Yang, M. Dao, S. Suresh, and T. J. Huang, Three-dimensional manipulation of single cells using surface acoustic waves, Proc. Natl. Acad. Sci. U.S.A. 113, 1522 (2016).

[16] A. Riaud, M. Baudoin, O. Bou Matar, J. L. Thomas, and P. Brunet, On the influence of viscosity and caustics on acoustic streaming in sessile droplets: an experimental and a numerical study with a cost-effective method, J. Fluid Mech. 821, 384 (2017).

[17] B. T. Hefner and P. L. Marston, An acoustical helicoidal wave transducer with applications for the alignment of ultrasonic and underwater systems, J. Acoust. Soc. Am. 106, 3313 (1999).

[18] J. L. Thomas and R. Marchiano, Pseudo Angular Momentum and Topological Charge Conservation for Nonlinear Acoustical Vortices, Phys. Rev. Lett. 91, 244302 (2003).

[19] R. Marchiano and J. L. Thomas, Doing Arithmetic With Nonlinear Acoustic Vortices, Phys. Rev. Lett. 101, 064301 (2008).

[20] A. Riaud, M. Baudoin, O. B. Matar, L. Becerra, and J. L. Thomas, Selective Manipulation of Microscopic Particles with Precursor Swirling Rayleigh Waves, Phys. Rev. Applied 7, 024007 (2017).

[21] A. Marzo, M. Caleap, and B. W. Drinkwater, Acoustic Virtual Vortices with Tunable Orbital Angular Momentum for Trapping of Mie Particles, Phys. Rev. Lett. 120, 044301 (2018).

[22] A. Marzo and B. W. Drinkwater, Holographic acoustic tweezers, Proc. Natl. Acad. Sci. U.S.A. (2018).

[23] W. J. Xie, C. D. Cao, Y. J. Lu, and B. Wei, Levitation of Iridium and Liquid Mercury by Ultrasound, Phys. Rev. Lett. 89, 104304 (2002).
[24] X. Ding, S. S. Lin, B. Kiralya, H. Yue, S. Li, I. K. Chiang, J. Shi, S. J. Benkovic, and T. J. Huang, On-chip manipulation of single microparticles, cells, and organisms using surface acoustic waves, Proc. Natl. Acad. Sci. U.S.A. 109, 11105 (2012).

[25] A. Ozcelik, J. Rufo, F. Guo, Y. Gu, P. Li, J. Lata, and T. J. Huang, Acoustic tweezers for the life sciences, Nat. Methods 15, 1021 (2018).

[26] K. C. Chitale, W. Presz, B. P. Ross-Johnsrud, M. Hyman, M. Lamontagne, and B. Lipkens, Particle manipulation using macroscale angled ultrasonic standing waves, Proc. Meet. Acoust. 30, 045004 (2017).

[27] D. Xu, F. Cai, M. Chen, F. Li, C. Wang, L. Meng, D. Xu, W. Wang, J. Wu, and H. Zheng, Acoustic manipulation of particles in a cylindrical cavity: theoretical and experimental study on the effects of boundary conditions, Ultrasonics 93, 18 (2019).

[28] P. B. Muller, R. Barnkob, M. J. H. Jensen, and H. Bruus, A numerical study of microparticle acoustophoresis driven by acoustic radiation forces and streaming-induced drag forces, Lab Chip 12, 4617 (2012).

[29] P. Hahn, I. Leibacher, T. Baasch, and J. Dual, Numerical simulation of acoustofluidic manipulation by radiation forces and acoustic streaming for complex particles, Lab Chip 15, 4302 (2015).

[30] S. Liu, Y. Yang, Z. Ni, X. Guo, L. Luo, J. Tu, D. Zhang, and J. Zhang, Investigation into the effect of acoustic radiation force and acoustic streaming on particle patterning in acoustic standing wave fields, Sensors 17, 1664 (2017).

[31] L. Zhang, Reversals of Orbital Angular Momentum Transfer and Radiation Torque, Phys. Rev. Applied 10, 034039 (2018).

[32] L. K. Zhang and P. L. Marston, Angular momentum flux of nonparaxial acoustic vortex beams and torques on axisymmetric objects, Phys. Rev. E 84, 065601 (2011).

[33] Z. Gong, P. L. Marston, W. Li, and Y. Chai, Multipole expansion of acoustical Bessel beams with arbitrary order and location, J. Acoust. Soc. Am. 141, EL574 (2017).

[34] G. T. Silva, Acoustic radiation force and torque on an absorbing compressible particle in an inviscid fluid, J. Acoust. Soc. Am. 136, 2405 (2014).

[35] J. P. Leão-Neto and G. T. Silva, Acoustic radiation force and torque exerted on a small viscoelastic particle in an ideal fluid, Ultrasonics 71, 1 (2016).

[36] J. H. Lopes, M. Azarpeyvand, and G. T. Silva, Acoustic interaction forces and torques acting on suspended spheres in an ideal fluid, IEEE Trans. Ultrason. Ferroelectr. Freq. Control 63, 186 (2016).

[37] D. Baresch, J. L. Thomas, and R. Marchiano, Orbital Angular Momentum Transfer to Stably Trapped Elastic Particles in Acoustical Vortex Beams, Phys. Rev. Lett. 121, 074301 (2018).

[38] R. Lim, A more stable transition matrix for acoustic target scattering by elongated objects, J. Acoust. Soc. Am. 138, 2266 (2015).

[39] Z. Gong, W. Li, F. G. Mitri, Y. Chai, and Y. Zhao, Arbitrary scattering of an acoustical Bessel beam by a rigid spheroid with large aspect-ratio, J. Sound Vibr. 383, 233 (2016).

[40] Z. Gong, W. Li, Y. Chai, Y. Zhao, and F. G. Mitri, T-matrix method for acoustical Bessel beam scattering from a rigid 
finite cylinder with spheroidal endcaps, Ocean Eng. 129, 507 (2017).

[41] W. Li, Y. Chai, Z. Gong, and P. L. Marston, Analysis of forward scattering of an acoustical zeroth-order Bessel beam from rigid complicated (aspherical) structures, J. Quant. Spectrosc. Radiat. Transf. 200, 146 (2017).

[42] Z. Gong, W. Li, and P. L. Marston, Potential of theoretical approach and T-matrix method for designing numerical acoustical tweezers toolbox, J. Acoust. Soc. Am. 143, 1739 (2018).

[43] Z. Gong, Ph.D. thesis, Huazhong University of Science and Technology, 2018.

[44] P. L. Marston, Scattering of a Bessel beam by a sphere, J. Acoust. Soc. Am. 121, 753 (2007).

[45] G. Maidanik, Torques due to acoustical radiation pressure, J. Acoust. Soc. Am. 30, 620 (1958).

[46] L. Zhang and P. L. Marston, Acoustic radiation torque and the conservation of angular momentum (L), J. Acoust. Soc. Am. 129, 1679 (2011).

[47] G. B. Arfken, H. J. Weber, and F. E. Harris, Mathematical Methods for Physicists (Elsevier, New York, 2013), 7th ed., Sec. 16.1, pp. 776-784.

[48] G. T. Silva, T. P. Lobo, and F. G. Mitri, Radiation torque produced by an arbitrary acoustic wave, Europhys. Lett. 97, 54003 (2012).

[49] B. Hartmann and J. Jarzynski, Immersion apparatus for ultrasonic measurements in polymers, J. Acoust. Soc. Am. 56, 1469 (1974).
[50] T. Hasegawa, Y. Hino, A. Annou, H. Noda, M. Kato, and N. Inoue, Acoustic radiation pressure acting on spherical and cylindrical shells, J. Acoust. Soc. Am. 93, 154 (1993).

[51] L. Flax, G. C. Gaunaurd, and H. Überall, Theory of resonance scattering, Phys. Acoust. 15, 191 (1981).

[52] G. C. Gaunaurd and M. F. Werby, Lamb and creeping waves around submerged spherical shells resonantly excited by sound scattering, J. Acoust. Soc. Am. 82, 2021 (1987).

[53] Z. Fan, D. Mei, K. Yang, and Z. Chen, Acoustic radiation torque on an irregularly shaped scatterer in an arbitrary sound field, J. Acoust. Soc. Am. 124, 2727 (2008).

[54] C. E. M. Démoré, Z. Yang, A. Volovick, S. Cochran, M. P. MacDonald, and G. C. Spalding, Mechanical Evidence of the Orbital Angular Momentum to Energy Ratio of Vortex Beams, Phys. Rev. Lett. 108, 194301 (2012).

[55] L. Zhang and P. L. Marston, Acoustic radiation torque on small objects in viscous fluids and connection with viscous dissipation, J. Acoust. Soc. Am. 136, 2917 (2014).

[56] P. Hahn, A. Lamprecht, and J. Dual, Numerical simulation of micro-particle rotation by the acoustic viscous torque, Lab Chip 16, 4581 (2015).

[57] S. G. Kargl and P. L. Marston, Observations and modeling of the backscattering of short tone bursts from a spherical shell: Lamb wave echoes, glory, and axial reverberations, J. Acoust. Soc. Am. 85, 1014 (1989).

[58] P. L. Marston, Acoustic beam scattering and excitation of sphere resonance: Bessel beam example, J. Acoust. Soc. Am. 122, 247 (2007); Erratum: 125, 4092 (2009). 